\title{
Collagen: Building Nature's Infrastructure
}

\author{
Russell M Jaffe* \\ Health Studies Collegium, Ashburn, VA, USA
}

*Corresponding author: Russell M Jaffe, CEO and Chairman of PERQUE Integrative Health (PIH), Fellow of Health Studies Collegium, Ashburn, VA 20147, USA.

Received Date: November 14, 2018

Published Date: November 26, 2018

\section{Collagen's Story}

Collagen has been a personal passion of mine since graduate school where my thesis tells the story of how collagen and elastin cross-links are affected by d-penicillamine, a remarkable amino acid. Collagen has been around since metazoan times. Its structure is elegantly simple and simply elegant. Glycine-proline-Any amino acid is a base unit that, when repeated about 1,000 times, becomes one strand of collagen. Three strands wind together to make a single collagen molecule. Collagen is a major part of the infrastructure of all mammals, fish, birds, and crustacean. The infrastructure includes bones and joints, arteries and veins, tendons and fascia. The orienting basement membrane that every cell receives nourishment from and exports metabolic products and wastes has a specialized collagen core.

\section{Acupuncture and Collagen Fibrils}

Acupuncture meridians or channels may actually be specialized collagen fibrils. The sentinel cells that emerge from the notochord in early gestation leave a collagen fibril as they migrate to all the systems of the body. Collagen is piezoelectric and this may be important in how acupuncture information is transferred so quickly throughout the body. Piezoelectric means that when compressed, a tiny yet measurable electrical current flows through that specific collagen fibril. The body's glycosaminoglycans functionally insulate and isolate collagen fibrils. Fibrils of collagen can be referred to as tactoids because they are narrow yet long. A silk thread is a metaphor for collagen. The fibrils are able to coil back on themselves and then stretch out depending on the biomechanical situation. If collagen is simply elegant ropes, elastin is diaphanous rubber bands. The cross-links in connective tissue are derived from the amino acid lysine. The epsilon amino group is removed. Aminoadipic acid semi-aldehyde is formed. From desmosines to lysinonorleucine, the cross-links are all derived from lysine. People who follow the Alkaline Way have diets that are naturally rich in alkaline amino acids such as lysine. Only in an alkaline cell environment is elective, essential protein like collagen produced.

\section{Collagen Renewal}

Renewal of collagen is the job of the immune defense and repair system. The innate immune responses are first line in regard to defending from foreign invasion and repairing worn out collagen or elastin. The immune system depends upon and uses up much cell energy. Adenosine triphosphate (ATP) is the currency of cell biology. The cell mitochondrial battery produces ATP only when there is enough cell magnesium to pair one magnesium electrolyte to each ATP. Magnesium deficit is increasingly common. Over the last half century, it is estimated that magnesium in America's food has declined by half at a time when need has doubled in part due to stress and environmental oxidative toxins. Enhanced uptake and chaperoned delivery of magnesium using choline citrate is work my group pioneered [1].

Youthful collagen is present when:

1. People take in fully buffered, fully reduced l-ascorbate (nature's vitamin C) based on their 'C Cleanse' self-assessment [2].

2. Polyphenolics, EPA and DHA omega 4 essential fats, complete $B$ complete, tocopherols and tocotrienols, selenomethione and Co Enzyme Q10 micellized in pure rice bran oil work as a synergistic team to enable cell energy. While supplementation was elective in the 20th century, the total allostatic and homeostic load of toxic matter in the 21st century requires comprehensive supplementation with evidence-based natural products.

3. From hatha and prana yoga to Tai Chi Chuan; from Feldenkrais and Anat Baniel Method to Trager and Integrative Myofascial Technique, there are body work techniques that promote gentle, deep relaxation while touch and stretch receptors in nerves are slowly stimulated.

4. A diet that can be digested, assimilated, and eliminated without immune burden is essential for a healthy digestive 
microbiome and healthy metabolome that is well nourished and has robust cell detoxification mechanisms.

5. Predictive Biomarker tests cover all of epigenetics; everything other than the DNA. Just eight tests meet the high standard as predictive markers. Predictive Biomarker tests are 'all cause morbidity and mortality' predicters. They have been done on enough people long enough to predict 10-year survival based on the value of the tests. The Predictive Biomarker tests are: Hemoglobin A1c, high sensitivity C-Reactive Protein, high sensitivity Homocysteine, high sensitivity Lymphocyte Response Assay, Urine pH after rest, Vitamin D levels, Omega 3 index, 8-oxoguanine. When interpreted to best outcome or goal value, disease risk can be identified long before catastrophe. People are resilient and energetic when each of their Predictive Biomarker tests is at its least risk value.

6. Staying hydrated and having a healthy digestive transit time are important in collagen renewal [3].

7. Added benefit comes from active meditation or similar mindfulness practice. Learned optimism and therapeutic biofeedback can also be helpful.

In contrast, taking collagen or gelatin supplements is a poor value and not helpful in renewing and rebuilding youthful collagen. Collagen protein is incomplete. It is not a quality protein. Collagen needs to be synthesized and put in place at the cell level. In addition, taking supplements of collagen or gelatin can be harsh on the kidneys. Taking collagen or gelatin supplements makes no sense to those who know biochemistry and systems biology. On the other hand, cheap protein sources go in and out of fashion. The Scarsdale Diet of the 1970s is an example of why people should not take collagen or gelatin supplements. Building collagen and connective tissue naturally is highly recommended.

\section{Collagen and d-Penicillamine}

Cross-links can be reversibly inhibited by d-penicillamine. This means the effect is present only if you take d-penicillamine (D-pen) on a regular, daily basis. On the other hand, d-penicillamine is a sulfur containing amino acid. Other names for the same molecule are mercaptovaline and dimethylcysteine. D-pen chelates toxic minerals such as lead, mercury, cadmium, arsenic, and nickel [4]. The d-pen complex is water soluble and removed safely from the body in urine, sweat and stool. The d-penicillamine protocol for quantifying all divalent cations is available including all nutritionally essential minerals and toxic minerals as well.

\section{Acknowledgement}

The author is CEO and owner of PERQUE Integrative Health.

\section{Conflict of Interest}

The author is CEO and owner of PERQUE Integrative Health.

\section{References}

1. Perque Integrative Health (2018) Addressing sleep issues in clinical practice: a discussion with Russell Jaffe, MD, PhD. Natural Medicine Journal 10(3).

2. Pinnel SR, Murad S, Darr D (1987) Induction of Collagen Synthesis by Ascorbic Acid: A Possible Mechanism. Arch Dermatol 123(12): 16841686.

3. Popkin BM, D’Anci KE, Rosenberg IH (2010) Water, Hydration and Health. Nutr Rev 68(8): 439-458.

4. Natalie S, Sisombath, Farideh Jalilehv, Adam C, Schell, et al. (2014) Lead (II) binding to the chelating agent C-penicillamine in aqueous solution. American Chemistry Society Inorganic Chemistry 53(23) :12459-12468. 